\title{
Molecular basis of the first cell fate determination in mouse embryogenesis
}

\author{
Lingyi Chen ${ }^{1}$, Dekun Wang ${ }^{1}$, Zhaoting $\mathrm{Wu}^{1}$, Liping $\mathrm{Ma}^{1}$, George Q Daley ${ }^{2,3,4,5,6}$ \\ ${ }^{\prime}$ Ministry of Education Key Laboratory of Bioactive Materials, College of Life Sciences, Nankai University, 94 Weijin Road, Tian- \\ jin 300071, China; ${ }^{2}$ Division of Pediatric Hematology/Oncology, Children's Hospital Boston and Dana Farber Cancer Institute, \\ 300 Longwood Avenue, Boston, MA 02115, USA; ${ }^{3}$ Department of Biological Chemistry and Molecular Pharmacology, Harvard \\ Medical School, Boston, MA, USA; ${ }^{4}$ Division of Hematology, Brigham and Women's Hospital, Boston, MA, USA; ${ }^{5}$ Harvard Stem \\ Cell Institute, Boston, MA, USA; ${ }^{6}$ Howard Hughes Medical Institute, Boston, MA, USA
}

Through proliferation and differentiation, a single cell, the zygote, can give rise to a complex organism composed of many types of cells. Up to the eight-cell embryo stage, the blastomeres are morphologically identical and distributed symmetrically in the mammalian embryo. Functionally, in some species, they are all totipotent. However, due to the compaction of blastomeres and the asymmetrical cell division at the late phase of the eight-cell embryo, the blastomeres of the morula are no longer identical. During the transition from morula to blastocyst, blastomeres differentiate, resulting in the first cell fate decision in embryogenesis, namely, the segregation of the inner cell mass and the trophectoderm. In this review, we will discuss the regulatory mechanisms essential for the cell fate choice during blastocyst development, including transcriptional regulation, epigenetic regulation, microRNAs, and signal transduction.

Keywords: inner cell mass; trophectoderm; embryogenesis

Cell Research (2010) 20:982-993. doi:10.1038/cr.2010.106; published online 13 July 2010

\section{Introduction}

A zygote can give rise to a complex organism through cell proliferation and differentiation. The first differentiation event in embryogenesis is the segregation of the trophectoderm (TE) and the inner cell mass (ICM) in the blastocyst. During the formation of the blastocyst, the zygote gradually loses its developmental potential. The zygote is totipotent, developing into not only the fetus but also the placenta. The totipotency is maintained in the blastomeres of the two-cell stage embryo. After mechanical separation of the blastomeres of the two-cell stage embryo, each blastomere is able to give rise to an adult mouse [1]. As the embryo develops further, the developmental potential of individual blastomere decreases. No
Correspondence: Lingyi Chen ${ }^{\mathrm{a}}$, George Q Daley ${ }^{\mathrm{b}}$

${ }^{\mathrm{a}}$ Tel: +86-22-2350-5821; Fax: +86-22-2350-5821

E-mail: lingyichen@nankai.edu.cn

${ }^{\mathrm{b}}$ Tel: +1-617-919-2013; Fax: +1-617-730-0222

E-mail: george.daley@childrens.harvard.edu mouse has been derived from a single blastomere at the four-cell stage or later $[2,3]$. Yet, when aggregated with 'carrier' blastomeres, single four- and eight-cell stage blastomeres are able to contribute to all the tissues of the embryo, suggesting that they are pluripotent [4]. Upon the formation of the blastocyst, cells in the embryo are differentiated into two distinct lineages, the multipotent TE and the pluripotent ICM. TE cells develop into the extraembryonic tissues, while ICM cells are able to give rise to the embryo proper and another extraembryonic tissue, the primitive endoderm (PE) [5]. How TE and PE form the fetal part of the placenta is described in more detail elsewhere [6].

Regarding the formation of the blastocyst, several questions of great biological importance remain unanswered or controversial. When and how do the cells become different from each other in the embryo? When and how are the fates of cells determined? How is the developmental potential of cells regulated during the transition from the totipotent zygote to the pluripotent ICM cells and the multipotent TE cells? In this review, we will focus on these questions, summarizing the recent progress 
in the field of early mouse embryogenesis.

\section{Developmental bias of the early blastomeres in mouse embryos}

Presently, when the segregation of the TE and the ICM begins remains controversial. Blastomeres in mouse embryos are morphologically identical up to the eight-cell stage. After compaction, blastomeres in the late eight-cell embryo are polarized. The first obvious differences between blastomeres appear during the transition from the 8 -cell to the 16-cell embryo, when cells are allocated to either inside or outside positions. The differentiation of ICM and TE appears to be initiated at this developmental stage. Yet, some data suggest that as early as the twocell stage, the blastomeres are biased toward either the TE or the ICM lineage, and the orientation and the order of the second cleavages correlate with blastocyst pattern in the mouse embryo. In about $80 \%$ of embryos, one of

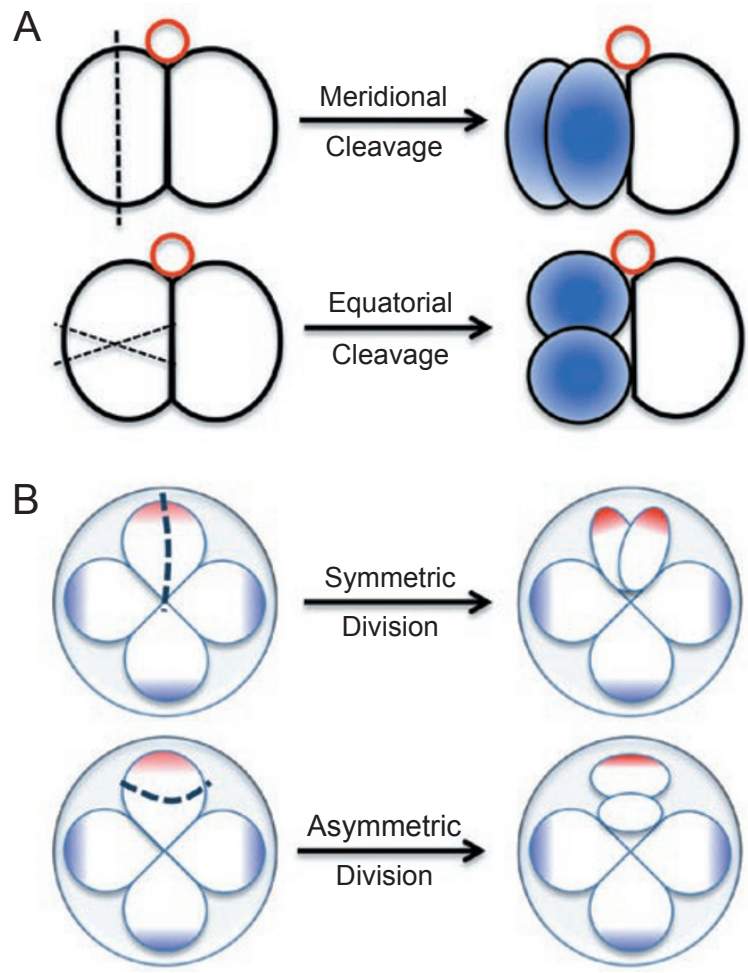

Figure 1 Schematic illustration for different types of cell division. (A) Equatorial (E) and meridional (M) cleavages in the two-cell embryo. The red circle is the second polar body. The dashed lines mark the cleavage planes. (B) Symmetric and asymmetric divisions in the eight-cell embryo. Only four blastomeres in the eight-cell embryo are shown. Symmetric division results in two polarized blastomeres with equal position in the embryo, while asymmetric division produces one polarized outer cell and one apolar inside cell. the second cleavages is equatorial or oblique (E), and the other is meridional (M) with respect to the second polar body (Figure 1A). These embryos can be divided into two groups, EM and ME embryos, according to the order of $\mathrm{E}$ and $\mathrm{M}$ cleavages. When the earlier of the second cleavage occurs meridionally (ME embryos), the progeny of the meridionally dividing cell populate predominantly the embryonic part of the blastocyst. In contrast, when the two-cell blastomere first divides equatorially or obliquely, the earlier equatorially dividing cell has an equal chance to develop into either the embryonic or the abembryonic part of the blastocyst. In the remaining $20 \%$ of embryos in which two-cell blastomeres undergo either EE or MM divisions, the contribution of progeny of the two-cell blastomeres is random, and developmental competence is compromised in EE and MM embryos [79]. The orientation and the order of the second cleavages might affect cell fate by asymmetric distribution of certain proteins. In four-cell ME embryos where pattern is predictable, the blastomeres derived from the $\mathrm{E}$ division tend to express more $\mathrm{Cdx} 2$, a TE-specific transcription factor [10]. Meanwhile, the M-derived blastomeres had higher levels of H3R26 methylation than the E-derived blastomeres [11]. How Cdx2 and H3R26 affect the cell fate choice is discussed in the sections on transcriptional and epigenetic regulation, respectively.

In contrast, other data argue that no strong developmental bias exists until the eight-cell stage. When the TE and ICM cells were traced back to the four-cell stage by in vitro time-lapse imaging, the majority of four-cell blastomeres gave rise to both embryonic and abembryonic regions. Only $7.6 \%$ and $8.3 \%$ of four-cell blastomeres contributed exclusively to the TE or the ICM, respectively. Furthermore, lineage tracing experiments demonstrated no such tendency for one of the two-cell blastomeres to contribute mainly to either the embryonic or abembryonic part of the blastocyst [12]. Therefore, it may be that some fractions of two-cell embryos have already segregated for TE and ICM, as in ME embryos, but in most cases, a developmental bias at this stage is insufficient to direct the ICM/TE lineage commitment. It has been suggested that embryo rotation within the zona pellucida and blastomere movement during cell division might change the location of blastomeres, hence, affecting cell fate commitment [12]. Certainly, complex events yet to be discovered are required to ensure proper TE and ICM differentiation.

\section{Cellular mechanism of allocating cells to the ICM or the TE}

TE cells are polarized epithelial cells on the surface of 
the blastocyst, while ICM cells are apolar cells in the inside of the blastocyst. Both cell polarity and cell position in the embryo play critical roles in initiating TE/ICM cell fate. Polarization of blastomeres begins at the late eightcell stage, as proteins including JAM1, aPKC, and PAR3, are localized at the apical membrane, and PAR1 becomes restricted to the basolateral regions [13, 14]. The polarity of the blastomeres results in two types of division, symmetric and asymmetric, during the transition from 8-cell to 16-cell embryos (Figure 1B). Symmetrical division results in two equally positioned and polarized cells, whereas asymmetrical division positions cells differentially. The outer cells retain polarity, and the inside cells inherit the basal pole of the parental cell, and hence lose polarity. The inside apolar cells tend to develop into the ICM, while the outside polarized cells tend to contribute to the TE [15]. In addition, cell polarity and cell position cross-regulate each other. When transplanted to outside positions, inside cells become polarized and adopt a TE fate [16-18]. Conversely, downregulation of polarity molecules, such as PAR3 and aPKC, promotes allocation of the cells to inside positions [13].

\section{The molecular basis underlying the segregation of the TE and the ICM}

As polarization of blastomeres occurs and the cells are allocated to either outside or inside positions, the specification of the TE and the ICM becomes apparent. Yet, even at the 16-cell stage, cells are not fully committed into the TE/ICM lineage. Manipulation of blastomeres at this stage may change cell fate, suggesting that 16-cell blastomeres remain plastic [19]. These blastomeres are able to sense environmental cues, which are transduced into cells through signaling pathways. Consequently, many changes within the cells, including transcriptional activity and epigenetic modifications, are directed by the signaling pathways to solidify the cell fate choice.

Cell fate determination is associated with changes in transcriptional profiles. Consequently, different sets of proteins are expressed to carry out distinct biological functions in various cells. Transcription of genes is regulated through many mechanisms, including transcription factors, epigenetic modifications, and microRNAs (miRNA). In addition, extracellular environmental cues can be transduced into intracellular compartments through signaling pathways, and regulate transcriptional activities.

\section{Transcriptional regulation in the TE/ICM specification}

During the development of the blastocyst, some transcription factors show restricted expression patterns as- sociated with the segregation of the ICM and the TE. For example, Nanog and Oct4 are expressed in ICM cells, but not in TE cells, while Cdx2 is exclusively expressed in the TE [20-22]. These transcription factors not only serve as markers for the ICM and the TE, but also play crucial roles in cell fate determination.

The homeodomain transcription factors Oct4 and Nanog were the first proteins identified as critical for both early embryo development and maintenance of pluripotency in embryonic stem (ES) cells [20, 21, 23]. Oct4 and Nanog are expressed in all blastomeres in the early morula, and become restricted to the ICM cells of blastocysts [23-26]. Both Oct4-null and Nanog-null embryos develop to the blastocyst stage, yet, no ES cells can be derived from them. Oct4-null ICM cells tend to differentiate along the extraembryonic trophoblast lineage, while Nanog-null ICM cells are limited to TE differentiation or apoptosis [20, 27], suggesting that Oct4-null and Nanog-null ICM cells are not pluripotent. Sox2, an HMG-box transcription factor, is also necessary for ICM formation. Like Oct4-null ICM, Sox2-null ICM cells divert into the trophoblast lineage after in vitro culture [28]. Sox 2 heterodimerizes with Oct 4 to regulate numerous genes in mouse ES cells [29]. Thus, loss of Sox2 might compromise Oct4 binding to its target genes, explaining why Sox2-null and Oct4-null ICMs show similar developmental defects.

In contrast, the development of the TE requires a distinct set of transcription factors, including Cdx2, Tead4, Eomes, and Elf5. Cdx2 and Tead4 act at an early stage for initial TE commitment, while Eomes and Elf5 reinforce the initial commitment to the trophoblast lineage [22, 30-33].

$C d x 2$-null blastocysts fail to implant, apparently due to inappropriate development of TE cells, as aberrant expression of Oct4 and Nanog can be detected in $C d x 2$-null TE cells [22]. Moreover, overexpression of $\mathrm{Cdx} 2$ in ES cells leads to differentiation toward the TE lineage [34]. Cdx2 may specify cells into the TE fate through several mechanisms. First, $\mathrm{Cdx} 2$ activates the transcription of TE genes. Second, $\mathrm{Cdx} 2$ promotes symmetric divisions at the eight-cell embryo stage, thus allocating more blastomeres to the outside TE. Third, Cdx2 affects cell polarity by enhancing aPKC expression. Consequently, polarized blastomeres are associated with asymmetric distribution of $\mathrm{Cdx} 2 \mathrm{mRNA}$. Therefore, $\mathrm{Cdx} 2$ and cellular polarity reinforce each other to make a firm commitment to the TE fate [10].

Tead4 is also necessary for TE formation. Deletion of Tead4 results in preimplantation lethality. Tead4null embryos fail to activate TE-specific genes, such as $C d x 2$, Eomes, and $F g f r 2$, while ICM markers, Nanog and 
Oct4, are expressed in all blastomeres. Moreover, no TS cells can be derived from Tead4-null embryos, whereas ES cell derivation is not affected $[30,31]$. These data indicate that Tead4 is essential for TE development. In addition, it has been demonstrated that the transcriptional activity of Tead4 is modulated by the cellular localization of its coactivator Yes-associated protein 1 (Yap1). Tead4 cooperates with unphosphorylated and nuclearlocalized Yap1 to activate Cdx2 and other TE genes [35]. Taken together, these data suggest that Tead4 acts upstream of $C d x 2$ in the specification of the TE lineage, but we cannot completely rule out the possibility that $\mathrm{Cdx} 2$ also regulates the expression of Tead4 and YAP to form a feed-forward regulatory loop.

Deletion of Eomes or Elf5 does not affect blastocyst formation and implantation, suggesting that they work downstream of $C d x 2$ and Tead4 in the TE development. Loss of Eomes prevents TE differentiation into trophoblast, resulting in embryonic lethality soon after implantation [32]. Deletion of Elf5 impairs the development of extraembryonic ectoderm [33].

Due to the importance of these lineage-specific transcription factors, their expression levels have to be tightly regulated. First, a feed-forward loop is formed among Nanog, Sox2, and Oct4 to maintain pluripotency [29, 3638]. In addition, Oct4 and Nanog are regulated by many other factors, such as Klf4, Zfx, Essrb, Smad1, STAT3, and Sall4 [38-40]. Second, ICM-specific and TE-specific transcription factors cross-regulate each other. Oct4 and Cdx2 occupy each other's promoter to suppress transcription. The antagonistic regulation between these two factors directs cells differentiating toward either the ICM or the TE lineage [34]. Similarly, cross-regulation of Nanog and Cdx2 also affects the segregation of the ICM and the TE [41]. Furthermore, a pluripotency-associated factor Sall4 not only regulates the ICM genes Oct4 and Nanog, but also suppresses TE gene expression. Knockdown or knockout of Sall4 in ES cells results in elevated Cdx2 expression [40, 42, 43]. Moreover, under the appropriate conditions, ES cells can be re-specified into the TE lineage by reducing Sall4 expression. Knockdown of Sall4 by siRNA microinjection into zygotes leads to aberrant Cdx2 expression in the ICM [40]. It has been shown that Sall4 binds to the $C d x 2$ promoter directly. Consequently, Sall4 may recruit histone deacetylase complex to inactivate $C d x 2$ transcription [43]. In addition, Sall4 activates the expression of Nanog and Oct4 by binding to their enhancer regions $[39,40]$. Thus, Sall4 might regulate $C d x 2$ expression through Nanog and Oct4. Despite Sall4's function in TE gene suppression, Sall4-null mutation does not affect lineage commitment in the blastocyst, as expression of lineage markers Oct4 and Cdx2 is not altered in Sall4-null embryos. Rather, Sall4 mutation impairs ICM proliferation, resulting in embryo death shortly after implantation [42].

Although many studies have focused on transcriptional regulation in ES and ICM cells, less is known about the transcription network in TE cells. Genetic studies reveal a hierarchy among the TE-specific factors: Tead4 is the most upstream factor, followed sequentially by Cdx2, Eomes, and Elf5 [22, 30-33]. In addition, GATA3 is involved in trophoblast development downstream of Tead4 and in parallel to Cdx2 [44]. Elevated expression of any one of these factors affects others, suggesting that they are regulating each other [34, 35, 45], but there is no strong biochemical evidence that these TE factors directly bind to each other's promoters. Regulation of $C d x 2$ is the best studied among these four factors. $\mathrm{Cdx} 2$ binds to the promoter and the intron 1 region of its own gene locus to activate itself [41]. GATA3 also upregulates $C d x 2$ expression by directly binding to the intron 1 region of the $C d x 2$ locus [46]. Other factors regulating the expression of these TE-specific factors remain to be identified.

Figure 2A illustrates the transcriptional regulation of TE/ICM differentiation, whereby lineage-specific factors positively regulate each other, and concomitantly suppress promoters of the alternate lineage, thereby reinforcing the cell fate commitment.

\section{Epigenetic regulation in the TE/ICM differentiation}

From the zygote to the formation of the blastocyst, the epigenome undergoes dramatic changes, such as DNA methylation, histone modification, and incorporation of histone variants $[47,48]$. The different epigenetic marks in blastomeres at various stages orchestrate the developmental potential of these blastomeres. The segregation of the TE and the ICM is also associated with asymmetric epigenetic marks [49].

Histone modifications Histone modification, including acetylation, methylation, phosphorylation, and ubiquitylation, is an important mechanism of epigenetic regulation [50]. The first wave of epigenetic reprogramming during preimplantation development occurs in the male pronucleus (PN). Upon fertilization, protamines associated with the paternal chromatin are rapidly replaced by acetylated histones, which are not methylated at H3K4, K9, or K27 [51]. Immediately after protamine-histone exchange, mono-methylation of $\mathrm{H} 3 \mathrm{~K} 4, \mathrm{~K} 9$, and $\mathrm{K} 27$ becomes detectable, implying that histones are rapidly deacetylated and mono-methylated [52]. Further methylation of mono-methylated histones is delayed. For example, $\mathrm{H} 3 \mathrm{~K} 4 \mathrm{me} 3$ is not present until the latest pronuclear stage $[52,53]$. While dynamic changes of histone 


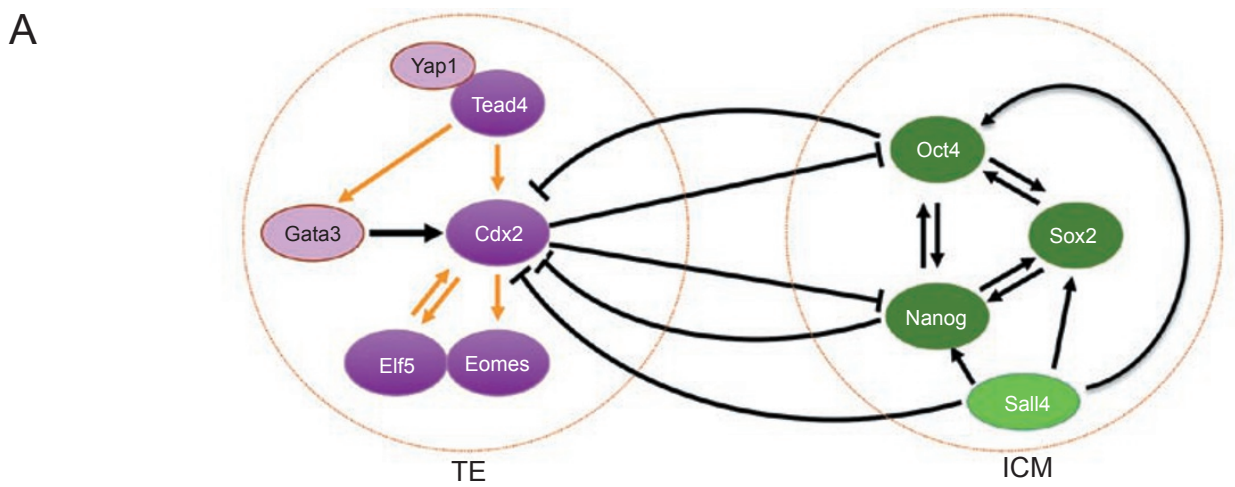

B

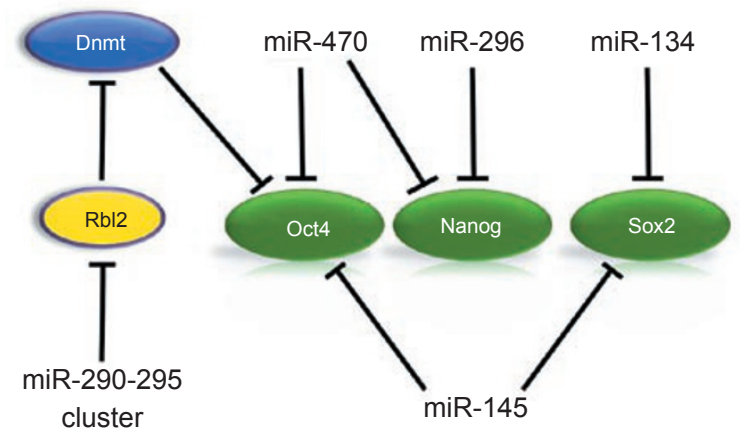

C

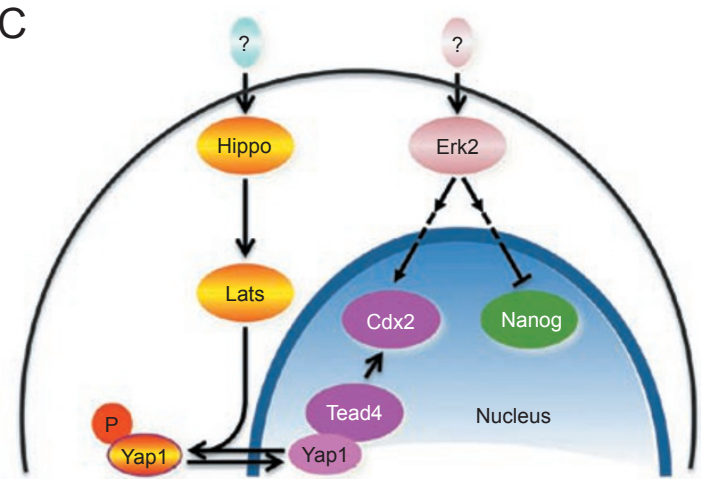

Figure 2 Molecular mechanisms for the ICM/TE segregation. (A) The regulatory network of key transcription factors in ICM/ TE specification. Cdx2, Tead4, Eomes, and Elf5 are the major TE-specific transcription factors. Tead4 cooperates with Yap1 to activate $C d x 2$, which in turn upregulates Eomes and Elf5. Conversely, Elf5 is able to enhance Cdx2 expression. In addition, GATA3 directly binds to the intron 1 region of the $C d x 2$ locus and activates $C d x 2$ expression. In contrast, Oct4, Nanog, and Sox 2 form a core regulatory circuitry to promote ICM cell fate. Sall4 not only activates Oct4, Nanog, and Sox2 but also suppresses $C d x 2$ expression, and this is critical to the differentiation of the ICM and the TE. Furthermore, cross-regulation between the ICM-specific factors and the TE-specific factors secures the appropriate cell fate in individual blastomeres. So far, no biochemical evidence demonstrates that Cdx2, Tead4, Eomes, and Elf5 bind to each other's promoters. To distinguish from other interactions with direct promoter binding, the genetic interactions among these four factors are marked with orange arrows. (B) MicroRNAs regulate the expression of pluripotency factors, Oct4, Nanog, and Sox2. Only the experimentally identified regulatory interactions are shown. (C) Two signaling pathways, the Hippo and MAPK pathways, are involved in the ICM/TE cell fate determination. In inside cells, Lats in the Hippo pathway phosphorylates Yap1, resulting in cytoplasmic distribution of Yap1. Without nuclear Yap1, Tead4 is unable to activate Cdx2. Thus, these cells develop to the ICM. In contrast, due to the inactive Hippo pathway in outer cell, Yap1 remains unphosphorylated and is localized in the nucleus. Yap1 cooperates with Tead4 to activate $C d x 2$, promoting the TE fate. Erk2 in the MAPK pathway activates Cdx2 and suppresses Nanog, facilitating the TE formation. Yet, the mechanisms for Erk2 regulating Cdx2 and Nanog, and the events upstream of Hippo and Erk2, remain unknown.

modifications occur in the male PN, maternal chromatin maintains all types of histone $\mathrm{H} 3$ methylation throughout zygotic development $[51,53,54]$. This reprogramming event might be essential for zygotic genome activation and establishment of totipotency.

During the formation of the blastocyst, the distinct TE and ICM lineages become marked by different histone modification profiles. Mono-, di-, and tri-methylation of H3K27 are more abundant in ICM cells than in TE cells, while methylation of $\mathrm{H} 3 \mathrm{~K} 9$ shows no significant difference between the TE and the ICM [55]. In addition to the global difference of H3K27 methylation, differential histone modifications are associated with specific loci in TE and ICM cells. For example, the active epigenetic marks, $\mathrm{H} 4 \mathrm{~K} 8 \mathrm{ac}$ and $\mathrm{H} 3 \mathrm{~K} 4 \mathrm{me} 3$, are more abundant at the promoters of Pou $5 f 1$ and Nanog in the ICM than in the TE. The repressive epigenetic mark $\mathrm{H} 3 \mathrm{~K} 9 \mathrm{me} 2$ is lacking in the promoters of Pou5f1 and Nanog in the ICM relative to the TE. In contrast, less H3K9me2 and more H4K8ac and $\mathrm{H} 3 \mathrm{~K} 4 \mathrm{me} 3$ at the $C d x 2$ promoter are found in TE cells relative to ICM cells [56]. Moreover, in ICM cells, $\mathrm{H} 4 \mathrm{~K} 16 \mathrm{ac}$ is relatively high at the promoters of Pouff1 
and Nanog, and is relatively low at the $C d x 2$ promoter [57]. All these histone modifications cooperate together to ensure the appropriate spatial and temporal expression of lineage-specific genes.

DNA methylation DNA methylation is another important epigenetic modification, normally associated with inactive genes. Following protamine replacement in the zygote, the DNA of the paternal genome is demethylated (except for some imprinted loci and repeat elements). This global demethylation event occurs before DNA replication, suggesting that this demethylation process is an active enzymatic reaction [58, 59]. It has been suggested that DNA deaminases might initiate the DNA demethylation reaction by deaminating $5 \mathrm{meC}$. Cytidine deamination mutates the $\mathrm{C}$ to $\mathrm{T}$; the $\mathrm{T}: \mathrm{G}$ mismatch is then repaired, resulting in replacement with an unmethylated cytidine [60]. Two recent papers have implicated the activation-induced cytidine deaminase AID in demethylation in the germ lineage, and in heterokaryons between ES cells and fibroblasts [61, 62]. The action of AID provides an appealing and plausible mechanism for demethylation, but further substantiation is required before acknowledging AID as the long-sought demethylase. Gadd45a has been shown to be involved in the repairmediated DNA demethylation [63]. A recent study has revealed that components of the elongator complex Elp1, Elp3, and Elp4 are essential for zygotic paternal genome demethylation [64]. In the opposite direction, PGC7/ Stella has been shown to protect maternal DNA from demethylation in early embryogenesis [65]. Thus, maternal DNA remains methylated while the paternal genome is rapidly demethylated at the zygote stage. In the subsequent divisions, the maternal genome is gradually demethylated $[59,66]$. The major cause for this demethylation is the absence of the maintenance methyltransferase Dnmt1 during DNA replication [67]. Through active and passive demethylation, respectively, paternal and maternal DNA methylation reaches a low level in the morula $[66,68]$. The global reprogramming of DNA methylation may cooperate with histone modifications to facilitate zygotic genome activation and to safeguard early embryo development.

From the morula to the blastocyst stage, global levels of methylation increase, yet unequally in the ICM and the TE. DNA methylation is greatly increased in the ICM, while TE cells remain relatively unmethylated despite an increment from the morula stage [59]. Proper DNA methylation levels in the ICM and the TE are critical for embryonic development. The loss of the maintenance methyltransferase Dnmt1 leads to severely retarded and abnormal development of the embryo proper, whereas development of extraembryonic tissues remains largely unaffected [49]. In addition, due to inefficient reprogramming, cloned embryos have high levels of DNA methylation. Particularly, TE cells in cloned embryos display aberrantly high levels of DNA methylation [69, 70], consistent with the observation that the prevailing developmental abnormality of cloned embryos is malformation of the placenta [49]. In summary, hypomethylation predominantly affects embryonic regulation and development rather than extraembryonic tissues, while hypermethylation significantly impairs epigenetic regulation and development of extraembryonic tissues.

X chromosome inactivation Another important epigenetic event in early embryogenesis is $\mathrm{X}$ chromosome inactivation (XCI) in female embryos. In each female mammalian cell, one of the two X chromosomes is transcriptionally inactivated to balance $X$-linked gene dosage between male (XY) and female (XX). After fertilization, the silencing of the paternal $\mathrm{X}$ chromosome starts at zygotic gene activation (two-cell stage) [71, 72]. Yet, how the paternal $\mathrm{X}$ chromosome is predominantly inactivated remains elusive. At the morula stage, the paternal X chromosome is inactivated in all cells. During the formation of the blastocyst, the inactive paternal X chromosome is reactivated in the epiblast, while TE and primitive endoderm cells maintain the silent status of the paternal $\mathrm{X}$ chromosome [72, 73]. After implantation, as epiblast cells further differentiate, the two X chromosomes undergo random inactivation [74].

XCI involves non-coding RNAs (ncRNAs), histone modifications, and DNA methylation. The ncRNA Xist is pivotal in XCI [75]. Upon initiation of XCI, Xist is transcribed from one of the two $\mathrm{X}$ chromosomes, and the resulting Xist RNA coats the X chromosome from which it is transcribed. The coating of Xist recruits factors responsible for transcriptional repression to the inactive $\mathrm{X}$ chromosome (Xi). Through the cooperation of these factors, the $\mathrm{Xi}$ is epigenetically marked with histone hypoacetylation and histone hypermethylation [76]. The expression of Xist is regulated by other ncRNAs, such as Tsix and Xite [77-79]. Yy1 and Ctcf have been characterized as trans-acting factors for both Xist and Tsix [80, 81]. More detailed mechanisms of XCI can be found in other review articles [76, 82].

While XCI has been studied extensively, little is known about the reactivation of the inactive paternal $\mathrm{X}$ chromosome in the ICM. Recently, it has been shown that the expression pattern of Nanog, but not Oct4 or Sox2, defines the ICM cells in which the $\mathrm{Xi}$ is reactivated, implying that Nanog might be involved in X chromosome reactivation. Moreover, Nanog-null ICMs fail to re- 
activate Xi [27]. Biochemical studies reveal that Nanog, Oct4, and Sox2 bind to the intronic region of Xist in ES cells $[38,83]$. In addition, Oct 4 and Sox 2 also occupy the Tsix and Xite loci [84]. It is possible that $\mathrm{Xi}$ reactivation is mediated by Xist suppression, and that the suppression of Xist in turn requires the cooperation of Nanog, Oct4, and Sox 2 . More investigations are necessary to better understand the mechanism of Xi reactivation. Recent success in the derivation of induced pluripotent stem (iPS) cells provides us an opportunity to survey the reactivation of $\mathrm{Xi}$ in vitro $[85,86]$.

Epigenetic regulation and developmental potential The differential epigenetic profiles of the ICM and the TE beg the question of which comes first: are the distinct epigenetic profiles a consequence of differentiation of the ICM and the TE, or do the differential epigenetic profiles precede lineage allocation and mediate the cell fate commitment? Intriguingly, uneven distribution of histone $\mathrm{H} 3$ with methylated arginine residues (R2, R17, and R26) is observed in the four-cell stage ME embryo, and the level of $\mathrm{H} 3$ methylation at these arginine residues (H3Rme) is correlated with cell fate and potency. Fourcell blastomeres with maximal H3Rme contribute more to the ICM and the polar TE, whereas blastomeres with minimal H3Rme contribute more to the mural TE. Moreover, overexpression of the H3-specific arginine methyltransferase CARM1 in individual blastomeres promotes their progeny to the ICM [11]. These data argue that epigenetic information precedes and dictates the ICM/ TE cell fate determination, by acting on critical lineagespecific genes. For example, overexpression of CARM1 enhances the expression levels of Nanog and Sox 2 in the injected blastomeres [11]. In ES cells, Oct4 recruits the histone H3K9 methyltransferase Eset to the $C d x 2$ promoter. Eset in turn catalyzes the methylation of $\mathrm{H} 3 \mathrm{~K} 9$ at the $C d x 2$ locus to suppress $C d x 2$ expression. Knockdown of Eset induces ES cell differentiation into the TE lineage $[87,88]$. Eset-depleted cells contribute to the TE of a blastocyst and, subsequently, placental tissues [88]. How epigenetic marks are first initiated remains a central question.

\section{miRNAs in the TE/ICM commitment}

miRNAs, a family of ncRNAs of 22 nucleotides, regulate gene expression through at least two distinct mechanisms: mRNA degradation and mRNA translational repression [89]. Given their important roles in gene regulation, miRNAs are also crucial for proper development, including early embryogenesis. Recent studies have revealed that miRNA expression profiles undergo dynamic changes during preimplantation embryo devel- opment, although significantly different miRNA profiles were observed with different methodologies [90-92]. The importance of miRNA in preimplantation embryo development is further supported by knockout of Dicer, an RNase III-family nuclease critical for RNA interference (RNAi) and miRNA generation. Loss of Dicer in mice results in embryonic death before E7.5, with a lack of detectable stem cells [93]. miRNAs are also important for ES cell differentiation. Dicer knockout ES cells show compromised proliferation and deficient differentiation $[94,95]$. Because both RNAi and miRNA generation are affected by Dicer knockout, it is not clear which pathway is responsible for the defect in ES cells. To assess the role of miRNAs in ES cells specifically, DGCR8, an RNA-binding protein that assists the RNase III enzyme Drosha in the processing of miRNA, was knocked out in mouse ES cells. Upon differentiation, DGCR8-deficient ES cells express some differentiation markers. However, pluripotency markers are not fully suppressed in DGCR8-deficient ES cells, confirming the crucial role of miRNAs in ES cell differentiation [96]. Altogether, these data suggest that miRNAs are essential for the differentiation of ICM and ES cells.

In addition, miRNAs might be involved in earlier stages of embryo development. To evaluate the functional importance of maternal miRNAs and Dicer, Dicer was conditionally knocked out in the growing oocyte, leading to defects in spindle organization and chromosomal alignment in the mature oocyte [90, 97]. When Dicer mutant oocytes were fertilized with wild-type sperm, the resulting zygotes failed to proceed through the first cell division [90]. Moreover, knockdown of Ago2, the catalytic component of the RNA-induced silencing complex, stabilizes one set of maternal RNAs and reduces zygotic transcripts of another set of genes, resulting in arrested embryos at the two-cell stage [98]. These data imply that miRNAs and/or siRNAs in the oocyte are essential for the earliest stages of embryogenesis. Further investigations are necessary to distinguish the specific effect of miRNAs and siRNAs.

Given the important role of miRNAs in embryogenesis, studies have been carried out to elucidate the function of individual miRNAs. By analyzing miRNA expression in undifferentiated and differentiated ES cells, a group of miRNAs have been found to be specifically expressed in undifferentiated ES cells, implying a role in maintenance of pluripotency. Conversely, another set of miRNAs is upregulated upon ES cell differentiation, indicating their functions in promoting differentiation [99]. Among the ES cell-specific miRNAs, the miR-290 cluster is of particular interest. The expression of the miR290 cluster begins at the four-cell stage, and steadily in- 
creases through the blastocyst stage, suggesting a critical role of the miR-290 cluster in early embryo development [92]. Mice with homozygous deletion of the miR-290 cluster die as embryos [100]. Furthermore, miR-291-3p, miR-294, and miR-295 enhance the derivation efficiency of mouse iPS cells by Oct4, Sox2, and Klf4, confirming their function in pluripotency establishment [101]. Similarly, miR-302 can reprogram human skin cancer cells into an ES-like pluripotent state [102]. In addition to the ES cell-specific miRNAs, a set of candidate miRNAs, which might be involved in TE specification, have also been identified, through comparing miRNA expression profiles in ES cell-derived TE cells and in staged murine embryos [92]. The functions of these miRNAs remain to be established experimentally.

To regulate gene expression, miRNAs bind to specific target mRNAs through imperfect match sequences. Hence, it is difficult to predict miRNA target genes. Nevertheless, several miRNAs have been demonstrated to regulate ICM-specific genes (Figure 2B). For example, miR-134 and miR470 target Sox 2 and Oct4, respectively. Both miR-296 and miR470 regulate Nanog expression. More importantly, the target sites of these miRNAs are located in the coding sequence, instead of the $3^{\prime}$ untranslated regions [103]. In human ES cells, miR-145 represses the expression of OCT4, SOX2, and KLF4 [104]. All these miRNAs negatively regulate pluripotency factors. It implies that these miRNAs might be involved in TE specification and ICM differentiation at a later stage. One target of the ES-specific miR-290 cluster has been identified. miRNAs of the miR-290 cluster suppress the expression of a Dnmt3 repressor Rbl2. Loss of the miR-290 cluster elevates Rb12 expression level, and in turn reduces Dnmt3 expression, resulting in DNA hypomethylation in ES cells and defect in stable Oct4 silencing after differentiation $[105,106]$. Thus, the miR-290 cluster plays important roles not only in pluripotency maintenance but also in ES cell differentiation and likely in ICM differentiation. So far, although a group of TE-specific miRNAs have been identified, none of their targets have been characterized in this context, and no miRNA regulators of TE-specific genes have been identified. Knowledge of these miRNAs will shed light on how miRNAs promote the segregation of TE and ICM.

\section{Signal transduction in TE/ICM segregation}

Blastomeres can relocate within the embryo during cell division or can be actively manipulated experimentally. In both scenarios, the final location of blastomeres determines the cell lineage, regardless of their original position, indicating that individual blastomeres can sense environmental cues and adopt the appropriate cell fate accordingly. To affect cell fate choice, the extracellular environmental cues must be transduced into intracellular compartments. Thus, signaling pathways play important roles in such a process.

Ras signaling appears to be essential for preimplantation embryo development. Inhibition of Ras by antisense oligonucleotides or monoclonal antibody does not affect normal oocyte maturation, but expression of the dominant-negative Ras N-17 mutant in fertilized eggs arrests the embryo at the two-cell stage. The inhibitory effect of Ras N-17 is overcome by simultaneous expression of an active raf oncogene. In contrast, expression of Ras N-17 starting from the late two-cell stage does not affect development of morula to blastocyst [107]. Thus, the Ras/ Raf signaling pathway is specifically required at the twocell stage.

Additionally, the MAPK pathways downstream of Ras are necessary for later stages of embryogenesis. The MAPK pathways include the extracellular signalregulated protein kinase (ERK) pathway, the Jun N-terminal kinase (JNK) pathway, and the p38 pathway. Early studies showed that inhibition of the JNK or the p38 pathway produced defects in blastocyst formation [108, 109]. Subsequently, more studies have revealed a role for the ERK pathway in blastocyst formation. It has been demonstrated that ectopic expression of an activated HRas $1^{\text {Q61L }}$ induced TE differentiation from ES cells. Erk2 is a major downstream effector of Ras for this cell fate switch. Interestingly, Erk2 is asymmetrically distributed in the apical membrane of the eight-cell embryo just before morula compaction. Inhibition of the ERK pathway in eight-cell embryos compromises $\mathrm{Cdx} 2$ expression, delays blastocyst development, and reduces TE outgrowth from embryo explants $[110,111]$. All these data imply that Erk2 might be involved in cell polarization, activation of $\mathrm{Cdx} 2$, and, consequently, in TE fate determination (Figure 2C). Furthermore, knockout of Erk2 results in early embryonic lethality, associated with defects in the ectoplacental cone and extraembryonic ectoderm, further confirming the crucial role of Erk2 in TE function [112].

In addition to the Ras-MAPK pathway, the recently discovered Hippo pathway is also implicated in ICM/TE differentiation $[35,113]$. Yap1 is a coactivator regulated by the Hippo pathway component Lats. In the mouse embryo, the phosphorylation status of Yap1 is correlated with the position of blastomeres, and regulates the cellular localization of Yap1 protein. In outside cells, unphosphorylated Yap1 is localized in the nucleus and cooperates with Tead4 to activate $C d x 2$. In inside cells, Yap1 becomes phosphorylated and cytoplasmic, hence downregulating the transcriptional activity of Tead4/ Yap1 complex [35]. Thus, through modulating the tran- 
scriptional activity of Tead4, the Hippo pathway facilitates the differentiation of the TE and the ICM (Figure 2C).

Isoforms of protein kinase $\mathrm{C}$ (PKC) might also be involved in the TE/ICM segregation. Activation of PKC leads to earlier compaction than normal embryos. Conversely, inhibitors of PKC, but not inhibitors of other kinases, block compaction [114]. PKC $\alpha$ is localized at the basolateral cortex adjacent to cell contact in 8-cell embryos, while $\mathrm{PKC} \mu$ is distributed to the outer cortex in 4-cell embryos, and to outside blastomeres in 16-cell embryos. $\mathrm{PKC} \xi$ also displays an asymmetric pattern in the four-cell embryo $[114,115]$. These results implicate PKC isotypes in the specification of the TE and the ICM through regulating cell polarization. However, the details of how PKC isotypes affect cell polarity remain unclear.

In many other situations, signaling pathways coordinately induce cellular responses to environmental cues [116]. It remains to be clarified whether and how the Ras-MAPK pathway, the Hippo pathway, and the PKC isotypes cross-regulate each other. Another important question is how the cell senses the outside/inside position and transduces this information to intracellular pathways.

\section{Conclusions}

The very first cell fate decision made in the early mammalian embryo - the segregation of ICM and TE remains a remarkably complex and little understood process. Changes of transcriptional profiles have pointed toward transcription factors as the key players. Oct4, Sox2, and Nanog form a regulatory circuit to promote ICM formation, whereas Tead 4 and $\mathrm{Cdx} 2$ operate to specify the TE. The establishment of new transcriptional profiles in distinct lineages is thereafter solidified by epigenetic modification and the action of miRNAs. The developmental potential of blastomeres is also tightly regulated through epigenetic modifications. Signaling pathways transduce environmental cues into the cells and affect gene expression, thus modulating cell fate determination.

Although our knowledge on the mechanisms of the ICM/TE segregation has grown considerably, there are still many questions unanswered. How do the key transcription factors interact with epigenetic regulators (such as histone-modifying enzymes) to activate or suppress gene expression? Which miRNAs are essential for blastocyst development? What are the critical targets of these miRNAs? How do blastomeres transduce extracellular signal into the intracellular signaling pathways? How do the Ras-MAPK, Hippo, and PKC pathways coordinately regulate cell fate commitment? These central questions constitute the challenge for the future.

\section{Acknowledgments}

GQD was supported by grants from the National Institutes of Health, the NIH Director's Pioneer Award of the NIH Roadmap for Medical Research, and private funds contributed to the Harvard Stem Cell Institute and the Children's Hospital Stem Cell Program, USA. GQD is a recipient of Clinical Scientist Awards in Translational Research from the Burroughs Wellcome Fund and the Leukemia and Lymphoma Society, and is an Investigator at the Howard Hughes Medical Institute. LC was supported by the National Natural Science Foundation of China (Grant no. 90919009), the National Key Basic Research and Development Program of China (Grant no. 2009CB941000 and 2010CB833603), and the Ministry of Agriculture of China (Grant no. 2009ZX08006-010B and 2009ZX08006-011B).

\section{References}

1 Tarkowski AK. Experiments on the development of isolated blastomers of mouse eggs. Nature 1959; 184:1286-1287.

2 Rossant J. Postimplantation development of blastomeres isolated from 4- and 8-cell mouse eggs. J Embryol Exp Morphol 1976; 36:283-290.

3 Tarkowski AK, Wroblewska J. Development of blastomeres of mouse eggs isolated at the 4- and 8-cell stage. J Embryol Exp Morphol 1967; 18:155-180.

4 Kelly SJ. Studies of the developmental potential of 4- and 8-cell stage mouse blastomeres. J Exp Zool 1977; 200:365376.

5 Ralston A, Rossant J. Genetic regulation of stem cell origins in the mouse embryo. Clin Genet 2005; 68:106-112.

6 Rossant J. Lineage development and polar asymmetries in the peri-implantation mouse blastocyst. Semin Cell Dev Biol 2004; 15:573-581.

7 Piotrowska-Nitsche K, Zernicka-Goetz M. Spatial arrangement of individual 4-cell stage blastomeres and the order in which they are generated correlate with blastocyst pattern in the mouse embryo. Mech Dev 2005; 122:487-500.

8 Piotrowska-Nitsche K, Perea-Gomez A, Haraguchi S, Zernicka-Goetz M. Four-cell stage mouse blastomeres have different developmental properties. Development 2005; 132:479490.

9 Bischoff M, Parfitt DE, Zernicka-Goetz M. Formation of the embryonic-abembryonic axis of the mouse blastocyst: relationships between orientation of early cleavage divisions and pattern of symmetric/asymmetric divisions. Development 2008; 135:953-962.

10 Jedrusik A, Parfitt DE, Guo G, et al. Role of Cdx2 and cell polarity in cell allocation and specification of trophectoderm and inner cell mass in the mouse embryo. Genes Dev 2008; 22:2692-2706.

11 Torres-Padilla ME, Parfitt DE, Kouzarides T, Zernicka-Goetz $\mathrm{M}$. Histone arginine methylation regulates pluripotency in the early mouse embryo. Nature 2007; 445:214-218.

12 Kurotaki Y, Hatta K, Nakao K, Nabeshima Y, Fujimori T. Blastocyst axis is specified independently of early cell lineage but aligns with the ZP shape. Science 2007; 316:719-723.

13 Plusa B, Frankenberg S, Chalmers A, et al. Downregulation of Par3 and aPKC function directs cells towards the ICM in 
the preimplantation mouse embryo. J Cell Sci 2005; 118:505515.

14 Thomas FC, Sheth B, Eckert JJ, Bazzoni G, Dejana E, Fleming TP. Contribution of JAM-1 to epithelial differentiation and tight-junction biogenesis in the mouse preimplantation embryo. J Cell Sci 2004; 117:5599-5608.

15 Yamanaka Y, Ralston A, Stephenson RO, Rossant J. Cell and molecular regulation of the mouse blastocyst. Dev Dyn 2006; 235:2301-2314.

16 Handyside AH. Time of commitment of inside cells isolated from preimplantation mouse embryos. J Embryol Exp Morphol 1978; 45:37-53.

17 Spindle AI. Trophoblast regeneration by inner cell masses isolated from cultured mouse embryos. J Exp Zool 1978; 203:483-489.

18 Rossant J, Lis WT. Potential of isolated mouse inner cell masses to form trophectoderm derivatives in vivo. Dev Biol 1979; 70:255-261.

19 Suwinska A, Czolowska R, Ozdzenski W, Tarkowski AK. Blastomeres of the mouse embryo lose totipotency after the fifth cleavage division: expression of $\mathrm{Cdx} 2$ and Oct 4 and developmental potential of inner and outer blastomeres of 16and 32-cell embryos. Dev Biol 2008; 322:133-144.

20 Nichols J, Zevnik B, Anastassiadis K, et al. Formation of pluripotent stem cells in the mammalian embryo depends on the POU transcription factor Oct4. Cell 1998; 95:379-391.

21 Mitsui K, Tokuzawa Y, Itoh H, et al. The homeoprotein Nanog is required for maintenance of pluripotency in mouse epiblast and ES cells. Cell 2003; 113:631-642.

22 Strumpf D, Mao CA, Yamanaka Y, et al. Cdx2 is required for correct cell fate specification and differentiation of trophectoderm in the mouse blastocyst. Development 2005; 132:20932102.

23 Chambers I, Colby D, Robertson M, et al. Functional expression cloning of Nanog, a pluripotency sustaining factor in embryonic stem cells. Cell 2003; 113:643-655.

24 Palmieri SL, Peter W, Hess H, Scholer HR. Oct-4 transcription factor is differentially expressed in the mouse embryo during establishment of the first two extraembryonic cell lineages involved in implantation. Dev Biol 1994; 166:259-267.

25 Dietrich JE, Hiiragi T. Stochastic patterning in the mouse preimplantation embryo. Development 2007; 134:4219-4231.

26 Hatano SY, Tada M, Kimura H, et al. Pluripotential competence of cells associated with Nanog activity. Mech Dev 2005; 122:67-79.

27 Silva J, Nichols J, Theunissen TW, et al. Nanog is the gateway to the pluripotent ground state. Cell 2009; 138:722-737.

28 Avilion AA, Nicolis SK, Pevny LH, Perez L, Vivian N, Lovell-Badge R. Multipotent cell lineages in early mouse development depend on SOX2 function. Genes Dev 2003; 17:126-140.

29 Boyer LA, Lee TI, Cole MF, et al. Core transcriptional regulatory circuitry in human embryonic stem cells. Cell 2005; 122:947-956.

30 Yagi R, Kohn MJ, Karavanova I, et al. Transcription factor TEAD4 specifies the trophectoderm lineage at the beginning of mammalian development. Development 2007; 134:38273836 .

31 Nishioka N, Yamamoto S, Kiyonari H, et al. Tead4 is required for specification of trophectoderm in pre-implantation mouse embryos. Mech Dev 2008; 125:270-283.

32 Russ AP, Wattler S, Colledge WH, et al. Eomesodermin is required for mouse trophoblast development and mesoderm formation. Nature 2000; 404:95-99.

33 Donnison M, Beaton A, Davey HW, Broadhurst R, L'Huillier $\mathrm{P}$, Pfeffer PL. Loss of the extraembryonic ectoderm in Elf5 mutants leads to defects in embryonic patterning. Development 2005; 132:2299-2308.

34 Niwa H, Toyooka Y, Shimosato D, et al. Interaction between Oct3/4 and $\mathrm{Cdx} 2$ determines trophectoderm differentiation. Cell 2005; 123:917-929.

35 Nishioka $\mathrm{N}$, Inoue $\mathrm{K}$, Adachi $\mathrm{K}$, et al. The Hippo signaling pathway components Lats and Yap pattern Tead4 activity to distinguish mouse trophectoderm from inner cell mass. Dev Cell 2009; 16:398-410.

36 Loh YH, Wu Q, Chew JL, et al. The Oct4 and Nanog transcription network regulates pluripotency in mouse embryonic stem cells. Nat Genet 2006; 38:431-440.

37 Kim J, Chu J, Shen X, Wang J, Orkin SH. An extended transcriptional network for pluripotency of embryonic stem cells. Cell 2008; 132:1049-1061.

38 Chen X, Xu H, Yuan P, et al. Integration of external signaling pathways with the core transcriptional network in embryonic stem cells. Cell 2008; 133:1106-1117.

39 Wu Q, Chen X, Zhang J, et al. Sall4 interacts with Nanog and co-occupies Nanog genomic sites in embryonic stem cells. $J$ Biol Chem 2006; 281:24090-24094.

40 Zhang J, Tam WL, Tong GQ, et al. Sall4 modulates embryonic stem cell pluripotency and early embryonic development by the transcriptional regulation of Pou5f1. Nat Cell Biol 2006; 8:1114-1123.

41 Chen L, Yabuuchi A, Eminli S, et al. Cross-regulation of the Nanog and Cdx2 promoters. Cell Res 2009; 19:1052-1061.

42 Sakaki-Yumoto M, Kobayashi C, Sato A, et al. The murine homolog of SALL4, a causative gene in Okihiro syndrome, is essential for embryonic stem cell proliferation, and cooperates with Sall1 in anorectal, heart, brain and kidney development. Development 2006; 133:3005-3013.

43 Yuri S, Fujimura S, Nimura K, et al. Sall4 is essential for stabilization, but not for pluripotency, of embryonic stem cells by repressing aberrant trophectoderm gene expression. Stem Cells 2009; 27:796-805.

44 Ralston A, Cox BJ, Nishioka N, et al. Gata3 regulates trophoblast development downstream of Tead4 and in parallel to Cdx2. Development 2010; 137:395-403.

$45 \mathrm{Ng}$ RK, Dean W, Dawson C, et al. Epigenetic restriction of embryonic cell lineage fate by methylation of Elf5. Nat Cell Biol 2008; 10:1280-1290.

46 Home P, Ray S, Dutta D, Bronshteyn I, Larson M, Paul S. GATA3 is selectively expressed in the trophectoderm of periimplantation embryo and directly regulates Cdx2 gene expression. J Biol Chem 2009; 284:28729-28737.

47 Morgan HD, Santos F, Green K, Dean W, Reik W. Epigenetic reprogramming in mammals. Hum Mol Genet 2005; 14 Spec No 1:R47-58.

48 Li E. Chromatin modification and epigenetic reprogramming in mammalian development. Nat Rev Genet 2002; 3:662-673.

49 Reik W, Santos F, Mitsuya K, Morgan H, Dean W. Epigenetic 
asymmetry in the mammalian zygote and early embryo: relationship to lineage commitment? Philos Trans $R$ Soc Lond $B$ Biol Sci 2003; 358:1403-1409.

50 Jenuwein T, Allis CD. Translating the histone code. Science 2001; 293:1074-1080.

51 Adenot PG, Mercier Y, Renard JP, Thompson EM. Differential $\mathrm{H} 4$ acetylation of paternal and maternal chromatin precedes DNA replication and differential transcriptional activity in pronuclei of 1-cell mouse embryos. Development 1997; 124:4615-4625.

52 Lepikhov K, Walter J. Differential dynamics of histone H3 methylation at positions $\mathrm{K} 4$ and $\mathrm{K} 9$ in the mouse zygote. BMC Dev Biol 2004; 4:12.

53 Santos F, Peters AH, Otte AP, Reik W, Dean W. Dynamic chromatin modifications characterise the first cell cycle in mouse embryos. Dev Biol 2005; 280:225-236.

54 Kourmouli N, Jeppesen P, Mahadevhaiah S, et al. Heterochromatin and tri-methylated lysine 20 of histone H4 in animals. J Cell Sci 2004; 117:2491-2501.

55 Erhardt S, Su IH, Schneider R, et al. Consequences of the depletion of zygotic and embryonic enhancer of zeste 2 during preimplantation mouse development. Development 2003; 130:4235-4248.

56 VerMilyea MD, O’Neill LP, Turner BM. Transcription-independent heritability of induced histone modifications in the mouse preimplantation embryo. PLoS One 2009; 4:e6086.

57 O'Neill LP, VerMilyea MD, Turner BM. Epigenetic characterization of the early embryo with a chromatin immunoprecipitation protocol applicable to small cell populations. Nat Genet 2006; 38:835-841.

58 Mayer W, Niveleau A, Walter J, Fundele R, Haaf T. Demethylation of the zygotic paternal genome. Nature 2000; 403:501502.

59 Santos F, Hendrich B, Reik W, Dean W. Dynamic reprogramming of DNA methylation in the early mouse embryo. Dev Biol 2002; 241:172-182.

60 Morgan HD, Dean W, Coker HA, Reik W, Petersen-Mahrt SK. Activation-induced cytidine deaminase deaminates 5-methylcytosine in DNA and is expressed in pluripotent tissues: implications for epigenetic reprogramming. $J$ Biol Chem 2004; 279:52353-52360.

61 Popp C, Dean W, Feng S, et al. Genome-wide erasure of DNA methylation in mouse primordial germ cells is affected by AID deficiency. Nature 2010; 463:1101-1105.

62 Bhutani N, Brady JJ, Damian M, Sacco A, Corbel SY, Blau HM. Reprogramming towards pluripotency requires AIDdependent DNA demethylation. Nature 2010; 463:1042-1047.

63 Barreto G, Schafer A, Marhold J, et al. Gadd45a promotes epigenetic gene activation by repair-mediated DNA demethylation. Nature 2007; 445:671-675.

64 Okada Y, Yamagata K, Hong K, Wakayama T, Zhang Y. A role for the elongator complex in zygotic paternal genome demethylation. Nature 2010; 463:554-558.

65 Nakamura T, Arai Y, Umehara H, et al. PGC7/Stella protects against DNA demethylation in early embryogenesis. Nat Cell Biol 2007; 9:64-71.

66 Rougier N, Bourc'his D, Gomes DM, et al. Chromosome methylation patterns during mammalian preimplantation development. Genes Dev 1998; 12:2108-2113.
67 Carlson LL, Page AW, Bestor TH. Properties and localization of DNA methyltransferase in preimplantation mouse embryos: implications for genomic imprinting. Genes Dev 1992; 6:2536-2541.

68 Howlett SK, Reik W. Methylation levels of maternal and paternal genomes during preimplantation development. Development 1991; 113:119-127.

69 Dean W, Santos F, Stojkovic M, et al. Conservation of methylation reprogramming in mammalian development: aberrant reprogramming in cloned embryos. Proc Natl Acad Sci USA 2001; 98:13734-13738.

70 Santos F, Zakhartchenko V, Stojkovic M, et al. Epigenetic marking correlates with developmental potential in cloned bovine preimplantation embryos. Curr Biol 2003; 13:11161121.

71 Huynh KD, Lee JT. Inheritance of a pre-inactivated paternal X chromosome in early mouse embryos. Nature 2003; 426:857-862.

72 Okamoto I, Otte AP, Allis CD, Reinberg D, Heard E. Epigenetic dynamics of imprinted $\mathrm{X}$ inactivation during early mouse development. Science 2004; 303:644-649.

73 Mak W, Nesterova TB, de Napoles M, et al. Reactivation of the paternal X chromosome in early mouse embryos. Science 2004; 303:666-669.

74 Monk M, Harper MI. Sequential X chromosome inactivation coupled with cellular differentiation in early mouse embryos. Nature 1979; 281:311-313.

75 Penny GD, Kay GF, Sheardown SA, Rastan S, Brockdorff N. Requirement for Xist in X chromosome inactivation. Nature 1996; 379:131-137.

76 Payer B, Lee JT. X chromosome dosage compensation: how mammals keep the balance. Annu Rev Genet 2008; 42:733772.

77 Lee JT, Davidow LS, Warshawsky D. Tsix, a gene antisense to Xist at the X-inactivation centre. Nat Genet 1999; 21:400404.

78 Lee JT, Lu N. Targeted mutagenesis of Tsix leads to nonrandom X inactivation. Cell 1999; 99:47-57.

79 Ogawa Y, Lee JT. Xite, X-inactivation intergenic transcription elements that regulate the probability of choice. $\mathrm{Mol}$ Cell 2003; 11:731-743.

80 Chao W, Huynh KD, Spencer RJ, Davidow LS, Lee JT. $\mathrm{CTCF}$, a candidate trans-acting factor for $\mathrm{X}$-inactivation choice. Science 2002; 295:345-347.

81 Donohoe ME, Zhang LF, Xu N, Shi Y, Lee JT. Identification of a Ctcf cofactor, Yy1, for the X chromosome binary switch. Mol Cell 2007; 25:43-56.

82 Heard E, Disteche CM. Dosage compensation in mammals: fine-tuning the expression of the X chromosome. Genes Dev 2006; 20:1848-1867.

83 Navarro P, Chambers I, Karwacki-Neisius V, et al. Molecular coupling of Xist regulation and pluripotency. Science 2008; 321:1693-1695.

84 Donohoe ME, Silva SS, Pinter SF, Xu N, Lee JT. The pluripotency factor Oct4 interacts with Ctcf and also controls X-chromosome pairing and counting. Nature 2009; 460:128132.

85 Takahashi K, Yamanaka S. Induction of pluripotent stem cells from mouse embryonic and adult fibroblast cultures by de- 
fined factors. Cell 2006; 126:663-676.

86 Stadtfeld M, Maherali N, Breault DT, Hochedlinger K. Defining molecular cornerstones during fibroblast to iPS cell reprogramming in mouse. Cell Stem Cell 2008; 2:230-240.

87 Yeap LS, Hayashi K, Surani MA. ERG-associated protein with SET domain (ESET)-Oct4 interaction regulates pluripotency and represses the trophectoderm lineage. Epigenetics Chromatin 2009; 2:12.

88 Yuan P, Han J, Guo G, et al. Eset partners with Oct4 to restrict extraembryonic trophoblast lineage potential in embryonic stem cells. Genes Dev 2009; 23:2507-2520.

89 Kloosterman WP, Plasterk RH. The diverse functions of microRNAs in animal development and disease. Dev Cell 2006; 11:441-450.

90 Tang F, Kaneda M, O'Carroll D, et al. Maternal microRNAs are essential for mouse zygotic development. Genes Dev 2007; 21:644-648.

91 Yang Y, Bai W, Zhang L, et al. Determination of microRNAs in mouse preimplantation embryos by microarray. Dev Dyn 2008; 237:2315-2327.

92 Viswanathan SR, Mermel CH, Lu J, Lu CW, Golub TR, Daley GQ. MicroRNA expression during trophectoderm specification. PLoS One 2009; 4:e6143.

93 Bernstein E, Kim SY, Carmell MA, et al. Dicer is essential for mouse development. Nat Genet 2003; 35:215-217.

94 Kanellopoulou C, Muljo SA, Kung AL, et al. Dicer-deficient mouse embryonic stem cells are defective in differentiation and centromeric silencing. Genes Dev 2005; 19:489-501.

95 Murchison EP, Partridge JF, Tam OH, Cheloufi S, Hannon GJ. Characterization of Dicer-deficient murine embryonic stem cells. Proc Natl Acad Sci USA 2005; 102:12135-12140.

96 Wang Y, Medvid R, Melton C, Jaenisch R, Blelloch R. DGCR8 is essential for microRNA biogenesis and silencing of embryonic stem cell self-renewal. Nat Genet 2007; 39:380385.

97 Murchison EP, Stein P, Xuan Z, et al. Critical roles for Dicer in the female germline. Genes Dev 2007; 21:682-693.

98 Lykke-Andersen K, Gilchrist MJ, Grabarek JB, Das P, Miska E, Zernicka-Goetz M. Maternal Argonaute 2 is essential for early mouse development at the maternal-zygotic transition. Mol Biol Cell 2008; 19:4383-4392.

99 Houbaviy HB, Murray MF, Sharp PA. Embryonic stem cellspecific MicroRNAs. Dev Cell 2003; 5:351-358.

100 Blakaj A, Lin H. Piecing together the mosaic of early mammalian development through microRNAs. J Biol Chem 2008; 283:9505-9508.

101 Judson RL, Babiarz JE, Venere M, Blelloch R. Embryonic stem cell-specific microRNAs promote induced pluripotency. Nat Biotechnol 2009; 27:459-461.

102 Lin SL, Chang DC, Chang-Lin S, et al. Mir-302 reprograms human skin cancer cells into a pluripotent ES-cell-like state. RNA 2008; 14:2115-2124.

103 Tay Y, Zhang J, Thomson AM, Lim B, Rigoutsos I. MicroRNAs to Nanog, Oct4 and Sox 2 coding regions modulate embryonic stem cell differentiation. Nature 2008; 455:11241128.

$104 \mathrm{Xu}$ N, Papagiannakopoulos T, Pan G, Thomson JA, Kosik KS. MicroRNA-145 regulates OCT4, SOX2, and KLF4 and represses pluripotency in human embryonic stem cells. Cell 2009; 137:647-658.

105 Benetti R, Gonzalo S, Jaco I, et al. A mammalian microRNA cluster controls DNA methylation and telomere recombination via Rb12-dependent regulation of DNA methyltransferases. Nat Struct Mol Biol 2008; 15:268-279.

106 Sinkkonen L, Hugenschmidt T, Berninger P, et al. MicroRNAs control de novo DNA methylation through regulation of transcriptional repressors in mouse embryonic stem cells. Nat Struct Mol Biol 2008; 15:259-267.

107 Yamauchi N, Kiessling AA, Cooper GM. The Ras/Raf signaling pathway is required for progression of mouse embryos through the two-cell stage. Mol Cell Biol 1994; 14:66556662.

108 Natale DR, Paliga AJ, Beier F, D’Souza SJ, Watson AJ. p38 MAPK signaling during murine preimplantation development. Dev Biol 2004; 268:76-88.

109 Maekawa M, Yamamoto T, Tanoue T, Yuasa Y, Chisaka O, Nishida E. Requirement of the MAP kinase signaling pathways for mouse preimplantation development. Development 2005; 132:1773-1783.

110 Lu CW, Yabuuchi A, Chen L, Viswanathan S, Kim K, Daley GQ. Ras-MAPK signaling promotes trophectoderm formation from embryonic stem cells and mouse embryos. Nat Genet 2008; 40:921-926.

111 Nichols J, Silva J, Roode M, Smith A. Suppression of Erk signalling promotes ground state pluripotency in the mouse embryo. Development 2009; 136:3215-3222.

112 Saba-El-Leil MK, Vella FD, Vernay B, et al. An essential function of the mitogen-activated protein kinase Erk2 in mouse trophoblast development. EMBO Rep 2003; 4:964-968.

113 Pan D. Hippo signaling in organ size control. Genes Dev 2007; 21:886-897.

114 Pauken CM, Capco DG. Regulation of cell adhesion during embryonic compaction of mammalian embryos: roles for PKC and beta-catenin. Mol Reprod Dev 1999; 54:135-144.

115 Pauken CM, Capco DG. The expression and stage-specific localization of protein kinase $\mathrm{C}$ isotypes during mouse preimplantation development. Dev Biol 2000; 223:411-421.

116 Guo X, Wang XF. Signaling cross-talk between TGF-beta/ BMP and other pathways. Cell Res 2009; 19:71-88. 